PROCEEDINGS OF THE

AMERICAN MATHEMATICAL SOCIETY

Volume 128, Number 11, Pages 3341-3347

S 0002-9939(00)05560-X

Article electronically published on May 11, 2000

\title{
A HIERARCHY OF THIN SETS RELATED TO THE BOUNDEDNESS OF TRIGONOMETRIC SERIES
}

\author{
PETER ELIAS̆
}

(Communicated by Christopher D. Sogge)

\begin{abstract}
We study the family $\mathcal{B}_{0}$ of the sets on which some series of the form $\sum_{k \in \mathbb{N}}\left|\sin \pi n_{k} x\right|$ is uniformly bounded. We show that the families $\mathcal{B}_{0}^{c}$ of all sets admitting the boundary $c$ form a hierarchy which is incontinuous with respect to the operations of intersection and union.
\end{abstract}

Let us recall two kinds of thin sets studied in harmonic analysis. A set $X \subseteq[0,1]$ is called an $N$-set (in honour of V. V. Nemytskii) if there exists a trigonometric series absolutely converging on $X$ but not converging absolutely everywhere; it is called an $N_{0}$-set if there exists a series of the form $\sum_{k \in \mathbb{N}}\left|\sin \pi n_{k} x\right|$ converging on $X$. J. Arbault [1] showed that the family $\mathcal{N}_{0}$ of all $\mathrm{N}_{0}$-sets is a proper subfamily of the family $\mathcal{N}$ of all $\mathrm{N}$-sets, and both families share some common properties.

In the paper [3] we examined several modifications of the definitions of the families $\mathcal{N}$ and $\mathcal{N}_{0}$, and compared the obtained families one to another. We showed that the family $\mathcal{B}_{0}$ of all sets on which some series of the form $\sum_{k \in \mathbb{N}}\left|\sin \pi n_{k} x\right|$ is uniformly bounded differs from previously known "classical" families. In the present paper we continue the study of the family $\mathcal{B}_{0}$ considering the hierarchy of the families $\mathcal{B}_{0}^{c}$ of all sets admitting the boundary $c$. We show that if $c<d$, then $\mathcal{B}_{0}^{c}$ is a proper subfamily of $\mathcal{B}_{0}^{d}$, and $\bigcup_{d<c} \mathcal{B}_{0}^{d} \varsubsetneqq \mathcal{B}_{0}^{c} \varsubsetneqq \bigcap_{d>c} \mathcal{B}_{0}^{d}$ for every $c>0$.

For a review of families of trigonometric thin sets, some historical notes, and also for many new results we refer the reader to the paper [2].

We shall deal with the quotient group $\mathbb{T}=\mathbb{R} / \mathbb{Z}$; however, we will not distinguish between the elements of $\mathbb{T}$ and the reals, or between the functions defined on $\mathbb{T}$ and the corresponding periodic functions on $\mathbb{R}$. For a real $x$, let $[x]$ denote the integer part of $x$ and let $\|x\|$ denote the distance of $x$ to the nearest integer, i.e. $[x]=$ $\max \{k \in \mathbb{Z}: k \leq x\}$ and $\|x\|=\min \{|x-k|: k \in \mathbb{Z}\}$. Let us note that $\|x\|-\|y\| \leq$ $\|x+y\| \leq\|x\|+\|y\|$ and $\|-x\|=\|x\|$ for all $x, y \in \mathbb{T}$. The space $\mathbb{T}$ equipped with the metric $\varrho(x, y)=\|x-y\|$ is a compact topological group.

In an accordance with [3], we define $\mathrm{B}_{0}$-sets as follows.

Definition 1. A set $X \subseteq \mathbb{T}$ is a $B_{0}$-set if there exists an increasing sequence $\left\{n_{k}\right\}_{k \in \mathbb{N}}$ of natural numbers such that the series $\sum_{k \in \mathbb{N}}\left\|n_{k} x\right\|$ is uniformly bounded

Received by the editors January 12, 1999.

2000 Mathematics Subject Classification. Primary 43A46; Secondary 42A05, 42A32.

Key words and phrases. Trigonometric thin sets, $\mathrm{N}_{0}$-sets, $\mathrm{B}_{0}$-sets, uniform boundedness.

This work was supported by grant 2/4034/97 of Slovak Grant Agency VEGA. The research was partly done when the author was visiting the Mathematical Institute of the University in Bonn with financial support by the Graduiertenkolleg. 
on $X$, i.e. there exists a real $c$ such that $\sum_{k \in \mathbb{N}}\left\|n_{k} x\right\| \leq c$ for all $x \in X$. The family of all $\mathrm{B}_{0}$-sets is denoted by $\mathcal{B}_{0}$.

Since $2\|x\| \leq|\sin \pi x| \leq \pi\|x\|$ for all $x \in \mathbb{T}$, it is clear that the previous definition remains equivalent if we replace $\sum_{k \in \mathbb{N}}\left\|n_{k} x\right\|$ by $\sum_{k \in \mathbb{N}}\left|\sin \pi n_{k} x\right|$.

Proposition 2. A set $X \subseteq \mathbb{T}$ is a $B_{0}$-set if and only if there exist an increasing sequence $\left\{n_{k}\right\}_{k \in \mathbb{N}}$ of natural numbers and a sequence $\left\{a_{k}\right\}_{k \in \mathbb{N}}$ of elements of $\mathbb{T}$ such that the series $\sum_{k \in \mathbb{N}}\left\|n_{k} x+a_{k}\right\|$ is uniformly bounded on $X$.

Proof. It follows immediately from Lemma 3 below.

Lemma 3. Let $\left\{n_{k}\right\}_{k \in \mathbb{N}}$ be an increasing sequence of natural numbers and let $\left\{a_{k}\right\}_{k \in \mathbb{N}}$ be a sequence of elements of $\mathbb{T}$. Then for every $\varepsilon>0$ there exists an increasing sequence $\left\{m_{j}\right\}_{j \in \mathbb{N}}$ of natural numbers such that

$$
\sum_{j \in \mathbb{N}}\left\|m_{j} x\right\| \leq \sum_{k \in \mathbb{N}}\left\|n_{k} x+a_{k}\right\|+\varepsilon
$$

for every $x \in \mathbb{T}$.

Proof. We use a classical argument. Since $\mathbb{T}$ is compact, there exists an increasing function $h: \mathbb{N} \rightarrow \mathbb{N}$ such that the sequence $\left\{n_{h(2 j+1)}-n_{h(2 j)}\right\}_{j \in \mathbb{N}}$ is increasing and $\sum_{j \in \mathbb{N}}\left\|a_{h(2 j+1)}-a_{h(2 j)}\right\| \leq \varepsilon$. It suffices to put $m_{j}=n_{h(2 j+1)}-n_{h(2 j)}$.

However, we do not know the answer to the following question.

Problem 4. Let $\left\{n_{k}\right\}_{k \in \mathbb{N}}$ be an increasing sequence of natural numbers and let $\left\{a_{k}\right\}_{k \in \mathbb{N}}$ be a sequence of elements of $\mathbb{T}$. Does there exist a sequence $\left\{m_{j}\right\}_{j \in \mathbb{N}}$ such that $\sum_{j \in \mathbb{N}}\left\|m_{j} x\right\| \leq \sum_{k \in \mathbb{N}}\left\|n_{k} x+a_{k}\right\|$ for all $x \in \mathbb{T}$ ?

Let us now define the families $\mathcal{B}_{0}^{c}$. Here the use of the series $\sum_{k \in \mathbb{N}}\left\|n_{k} x\right\|$ and $\sum_{k \in \mathbb{N}}\left|\sin \pi n_{k} x\right|$ is not equivalent; for simplicity we will consider the first one.

Definition 5. Let $c$ be a positive real. A set $X \subseteq \mathbb{T}$ is called a $B_{0}^{c}$-set if there exists an increasing sequence $\left\{n_{k}\right\}_{k \in \mathbb{N}}$ of natural numbers such that $\sum_{k \in \mathbb{N}}\left\|n_{k} x\right\| \leq c$ for all $x \in X$. The family of all $\mathrm{B}_{0}^{c}$-sets is denoted by $\mathcal{B}_{0}^{c}$.

We show that in the previous definition ' $\leq$ ' can be replaced by ' $<$ '.

Lemma 6. Let $c>0, X \subseteq \mathbb{T}$, and let $\left\{n_{k}\right\}_{k \in \mathbb{N}}$ be such that $\sum_{k \in \mathbb{N}}\left\|n_{k} x\right\| \leq c$ for all $x \in X$. Then there exists $j \in \mathbb{N}$ such that $\sum_{k>j}\left\|n_{k} x\right\|<c$ for all $x \in X$.

Proof. Suppose for a contradiction that for every $j$ there exists $x_{j} \in X$ such that $\sum_{k>j}\left\|n_{k} x_{j}\right\| \geq c$. Thus $\left\|n_{k} x_{j}\right\|=0$ for all $k \leq j$ and $\left\|n_{k_{j}} x_{j}\right\|>0$ for some $k_{j}>j$. Hence for every $j$ and $k$ such that $k \geq k_{j}$ we have $\left\|n_{k_{j}} x_{j}\right\|>0$ and $\left\|n_{k_{j}} x_{k}\right\|=0$, and thus $x_{j} \neq x_{k}$. It follows that the set $\left\{x_{j}: j \in \mathbb{N}\right\}$ is infinite. However, this is impossible since for every $k$ there are only finitely many $x \in \mathbb{T}$ such that $\left\|n_{k} x\right\|=0$.

Let us recall that a set $X \subseteq \mathbb{T}$ is called a Dirichlet set (a D-set) if there exists an increasing sequence $\left\{n_{k}\right\}_{k \in \mathbb{N}}$ of natural numbers such that the sequence $\left\{\left\|n_{k} x\right\|\right\}_{k \in \mathbb{N}}$ is uniformly converging to 0 on $X$. The family of all $\mathrm{D}$-sets is denoted by $\mathcal{D}$. From the definition we can immediately see that $\bigcup_{c>0} \mathcal{B}_{0}^{c}=\mathcal{B}_{0}$ and $\bigcap_{c>0} \mathcal{B}_{0}^{c}=\mathcal{D}$.

We do not know whether an analogue of Proposition 2 holds true in the case of $\mathrm{B}_{0}^{c}$-sets. 
Problem 7. Let $c>0$, let $\left\{n_{k}\right\}_{k \in \mathbb{N}}$ be an increasing sequence of natural numbers and let $\left\{a_{k}\right\}_{k \in \mathbb{N}}$ be a sequence of elements of $\mathbb{T}$. Does there exist a sequence $\left\{m_{j}\right\}_{j \in \mathbb{N}}$ such that for all $x \in \mathbb{T}, \sum_{j \in \mathbb{N}}\left\|m_{j} x\right\| \leq c$ whenever $\sum_{k \in \mathbb{N}}\left\|n_{k} x+a_{k}\right\| \leq c$ ?

Fix a positive real $c$. Let us denote by $\mathcal{B}_{0}^{* c}$ the family of all sets $X \subseteq \mathbb{T}$ for which there exist sequences $\left\{n_{k}\right\}_{k \in \mathbb{N}}$ and $\left\{a_{k}\right\}_{k \in \mathbb{N}}$ such that $\sum_{k \in \mathbb{N}}\left\|n_{k} x+a_{k}\right\| \leq c$ for all $x \in X$. Clearly $\mathcal{B}_{0}^{c} \subseteq \mathcal{B}_{0}^{* c}$, and Problem $[7$ asks whether the equality holds true. From Lemma 3 it follows that $\mathcal{B}_{0}^{* c} \subseteq \bigcap_{d>c} \mathcal{B}_{0}^{d}$. Thus for every $c>0$ we have

$$
\bigcup_{d<c} \mathcal{B}_{0}^{d}=\bigcup_{d<c} \mathcal{B}_{0}^{* d} \subseteq \mathcal{B}_{0}^{c} \subseteq \mathcal{B}_{0}^{* c} \subseteq \bigcap_{d>c} \mathcal{B}_{0}^{d}=\bigcap_{d>c} \mathcal{B}_{0}^{* d} .
$$

In Propositions 10 and 11 we will show that the first and the last inclusions are proper.

Let $\left\{m_{j}\right\}_{j \in \mathbb{N}}$ be an increasing sequence of natural numbers and let $\left\{a_{j}\right\}_{j \in \mathbb{N}}$ be a sequence of elements of $\mathbb{T}$. For every positive integer $n$, let $\alpha(n), \beta(n) \in \mathbb{N}$ be such that $n=2^{\alpha(n)} \beta(n)$ and $\beta(n)$ is odd.

Lemma 8. Let $p, r \in \mathbb{N}, p \geq 1$. Assume that the set $\left\{\alpha\left(m_{j}\right): j \in \mathbb{N}\right\}$ is infinite. There exist $j, q \in \mathbb{N}$ and $s \in\{-1,1\}$ such that $q \geq r$ and $\left\|s m_{j} 2^{-q 2^{p}}+a_{j}\right\| \geq 2^{-2^{p}}$.

Proof. Put $J=\left\{j \in \mathbb{N}:\left\|a_{j}\right\| \geq 2^{-2^{p}}\right\}$. Clearly either $\left\{\alpha\left(m_{j}\right): j \in J\right\}$ or $\left\{\alpha\left(m_{j}\right)\right.$ : $j \notin J\}$ is infinite.

In the first case find $j \in J$ such that $\alpha\left(m_{j}\right) \geq r 2^{p}$, and put $q=r$ and $s=1$. Then $s m_{j} 2^{-q 2^{p}}$ is an integer and thus $\left\|s m_{j} 2^{-q 2^{p}}+a_{j}\right\|=\left\|a_{j}\right\| \geq 2^{-2^{p}}$.

In the latter case find $j \notin J$ such that $\alpha\left(m_{j}\right) \geq(r-1) 2^{p}$ and put $q=$ $\left[\alpha\left(m_{j}\right) 2^{-p}\right]+1$. Then clearly $q \geq r$. We also have $0<q 2^{p}-\alpha\left(m_{j}\right) \leq 2^{p}$ and thus $\left\|m_{j} 2^{-q 2^{p}}\right\|=\left\|\beta\left(m_{j}\right) 2^{\alpha\left(m_{j}\right)-q 2^{p}}\right\| \geq 2^{-2^{p}}$. Since $\left\|a_{j}\right\| \leq 2^{-2^{p}} \leq 2^{-2}$, we can conclude that either $\left\|m_{j} 2^{-q 2^{p}}+a_{j}\right\| \geq 2^{-2^{p}}$ or $\left\|m_{j} 2^{-q 2^{p}}-a_{j}\right\| \geq 2^{-2^{p}}$.

Lemma 9. Let $\theta$ be a real such that $0<\theta \leq 1 / 2$, let $\left\{p_{k}\right\}_{k \in \mathbb{N}}$ be a sequence of positive integers, and let $\left\{\varepsilon_{k}\right\}_{k \in \mathbb{N}}$ be a sequence of positive reals. Assume that $\lim _{j \rightarrow \infty} \alpha\left(m_{j}\right)=\infty$. There exists $x \in \mathbb{T}$ such that

(1) $\|x\| \leq \theta$;

(2) there exists an increasing sequence $\left\{n_{k}\right\}_{k \in \mathbb{N}}$ of natural numbers such that $\left\|2^{2^{n_{k}}} x\right\| \leq 2^{-2^{p_{k}}}+2^{-2^{n_{k}}}$ for all $k \in \mathbb{N}$, and $\left\|2^{2^{n}} x\right\| \leq 2^{-2^{n}}$ for all $n \notin$ $\left\{n_{k}: k \in \mathbb{N}\right\}$

(3) there exists an increasing sequence $\left\{j_{k}\right\}_{k \in \mathbb{N}}$ of natural numbers such that $\left\|m_{j_{k}} x+a_{j_{k}}\right\| \geq 2^{-2^{p_{k}}}-\varepsilon_{k}$ for all $k \in \mathbb{N}$.

Proof. We will define sequences $\left\{n_{k}\right\}_{k \in \mathbb{N}},\left\{j_{k}\right\}_{k \in \mathbb{N}},\left\{q_{k}\right\}_{k \in \mathbb{N}}$, and $\left\{s_{k}\right\}_{k \in \mathbb{N}}$ by induction. Fix $k \in \mathbb{N}$ and suppose that $n_{i}, j_{i}, q_{i}, s_{i}$ are defined for all $i<k$. Find $r \geq 2$ such that

(a) $2^{-r 2^{p_{k}}} \leq 2^{-k-1} \theta$;

(b) $r 2^{p_{k}}>2^{n_{k-1}+1}$ if $k \geq 1$;

(c) $2^{-r 2^{p_{k}}} \leq 2^{i-k} 2^{-2^{n_{i}+1}}$ for all $i<k$;

(d) $2^{-r 2^{p_{k}}} \leq 2^{i-k} \varepsilon_{i} / m_{j_{i}}$ for all $i<k$.

Let $l \in \mathbb{N}$ be such that $l>j_{k-1}$ if $k \geq 1$, and $\alpha\left(m_{j}\right) \geq q_{k-1} 2^{p_{k-1}}$ for all $j \geq l$. By Lemma 8 there exist $j_{k} \geq l, q_{k} \geq r$, and $s_{k} \in\{-1,1\}$ such that

(e) $\left\|m_{j_{k}} s_{k} 2^{-q_{k} 2^{-p_{k}}}+a_{j_{k}}\right\| \geq 2^{-2^{p_{k}}}$. 
Put $n_{k}=\max \left\{i: 2^{i}<q_{k} 2^{p_{k}}\right\}$.

Let $x=\sum_{k \in \mathbb{N}} s_{k} 2^{-q_{k} 2^{p_{k}}}$. By (a), $|x| \leq \sum_{k \in \mathbb{N}} 2^{-q_{k} 2^{p_{k}}} \leq \sum_{k \in \mathbb{N}} 2^{-k-1} \theta=\theta$, and thus (1) holds.

To show (2), fix $k \in \mathbb{N}$. If $k \geq 1$, then by (b), $q_{k} 2^{p_{k}}>2^{n_{k-1}+1}$. Thus $n_{k}>n_{k-1}$ and hence $q_{i} 2^{p_{i}}<2^{n_{k}}$ for all $i<k$. Since $q_{k} \geq 2$, we have $2^{n_{k}+1} \geq q_{k} 2^{p_{k}} \geq 2^{p_{k}+1}$ and thus $n_{k} \geq p_{k}$. Hence $2^{n_{k}}$ is a multiple of $2^{p_{k}}$ and we have $q_{k} 2^{p_{k}} \geq 2^{n_{k}}+2^{p_{k}}$. Using (c) we obtain that

$$
\begin{aligned}
\sum_{i \geq k} 2^{-q_{i} 2^{p_{i}}} & \leq 2^{-q_{k} 2^{p_{k}}}+\sum_{i>k} 2^{k-i} 2^{-2^{n_{k}+1}} \\
& \leq 2^{-2^{n_{k}}-2^{p_{k}}}+2^{-2^{n_{k}+1}}=2^{-2^{n_{k}}}\left(2^{-2^{p_{k}}}+2^{-2^{n_{k}}}\right) .
\end{aligned}
$$

Hence $\left\|2^{2^{n_{k}}} x\right\| \leq 2^{2^{n_{k}}} \sum_{i \geq k} 2^{-q_{i} 2^{p_{i}}} \leq 2^{-2^{p_{k}}}+2^{-2^{n_{k}}}$, and thus the first part of (2) holds. To show the latter one, let $n \notin\left\{n_{k}: k \in \mathbb{N}\right\}$ and put $k=\min \left\{i: n_{i}>n\right\}$. Then for all $i<k$ we have $n>n_{i}$ and thus $q_{i} 2^{p_{i}} \leq 2^{n}$. Hence by (f),

$$
\begin{aligned}
\left\|2^{2^{n}} x\right\| & \leq 2^{2^{n}} \sum_{i \geq k} 2^{-q_{2} 2^{p_{i}}} \\
& \leq 2^{2^{n}-2^{n_{k}}}\left(2^{-2^{p_{k}}}+2^{-2^{n_{k}}}\right) \leq 2^{2^{n}-2^{n+1}}=2^{-2^{n} .}
\end{aligned}
$$

To show (3), fix again $k \in \mathbb{N}$. From the choice of $l$ it follows that $j_{k}>j_{k-1}$ if $k \geq 1$, and $\alpha\left(m_{j_{k}}\right) \geq q_{i} 2^{p_{i}}$ for all $i<k$. Hence by (e) and (d),

$$
\begin{aligned}
\left\|m_{j_{k}} x+a_{j_{k}}\right\| & \geq\left\|m_{j_{k}} s_{k} 2^{-q_{k} 2^{p_{k}}}+a_{j_{k}}\right\|-m_{j_{k}} \sum_{i>k} 2^{-q_{i} 2^{p_{i}}} \\
& \geq 2^{-2^{p_{k}}}-m_{j_{k}} \sum_{i>k} 2^{k-i} \varepsilon_{k} / m_{j_{k}}=2^{-2^{p_{k}}}-\varepsilon_{k} .
\end{aligned}
$$

For $n \in \mathbb{N}$, denote $\phi(n)=\sum_{k>n} 2^{-2^{k}}, \psi(0)=1$, and $\psi(n)=2^{-2^{n}} \sum_{k<n} 2^{2^{k}}$ if $n \geq 1$. It can be easily shown that both $\{\phi(n)\}_{n \in \mathbb{N}}$ and $\{\psi(n)\}_{n \in \mathbb{N}}$ are decreasing and converging to 0 .

Proposition 10. For every $c>0$ there exists $X \subseteq \mathbb{T}$ such that

(1) there exists an increasing sequence $\left\{n_{k}\right\}_{k \in \mathbb{N}}$ of natural numbers such that $\sum_{k \in \mathbb{N}}\left\|n_{k} x\right\| \leq c$ for all $x \in X$

(2) for every increasing sequence $\left\{m_{j}\right\}_{j \in \mathbb{N}}$ of natural numbers, every sequence $\left\{a_{j}\right\}_{j \in \mathbb{N}}$ of elements of $\mathbb{T}$, and for every $\eta>0$ there exists $x \in X$ such that $\sum_{j \in \mathbb{N}}\left\|m_{j} x+a_{j}\right\|>c-\eta$.

Proof. Put $X=\left\{x \in \mathbb{T}: \sum_{n \in \mathbb{N}}:\left\|2^{2^{n}} x\right\| \leq c\right\}$. Clearly $X$ satisfies the condition (1).

To show (2), fix $\eta>0$ and sequences $\left\{m_{j}\right\}_{j \in \mathbb{N}},\left\{a_{j}\right\}_{j \in \mathbb{N}}$. We will find $x \in X$ such that $\sum_{j \in \mathbb{N}}\left\|m_{j} x+a_{j}\right\|>c-\eta$. We will consider two cases.

(a) If $\lim _{j \rightarrow \infty} \alpha\left(m_{j}\right)=\infty$, then we can find $N \in \mathbb{N}$, a sequence $\left\{p_{k}\right\}_{k \in \mathbb{N}}$ of positive integers and a sequence $\left\{\varepsilon_{k}\right\}_{k \in \mathbb{N}}$ of positive reals such that $\sum_{k \in \mathbb{N}} 2^{-2^{p} k}-$ $\sum_{k \in \mathbb{N}} \varepsilon_{k}>c-\eta$ and $\sum_{k \in \mathbb{N}} 2^{-2^{p_{k}}}+\phi(N)+\psi(N) \leq c$. Put $\theta=2^{-2^{N}}$. There exists 
$x \in \mathbb{T}$ satisfying the conditions (1)-(3) of Lemma 9. We have

$$
\sum_{n \in \mathbb{N}}\left\|2^{2^{n}} x\right\|=\sum_{n<N}\left\|2^{2^{n}} x\right\|+\sum_{n \geq N}\left\|2^{2^{n}} x\right\| \leq \sum_{n<N} 2^{2^{n}} \theta+\sum_{k \in \mathbb{N}} 2^{-2^{p_{k}}}+\sum_{n \geq N} 2^{-2^{n}} \leq c
$$

and

$$
\sum_{j \in \mathbb{N}}\left\|m_{j} x+a_{j}\right\| \geq \sum_{k \in \mathbb{N}}\left\|m_{j_{k}} x+a_{j_{k}}\right\| \geq \sum_{k \in \mathbb{N}}\left(2^{-2^{p_{k}}}-\varepsilon_{k}\right)>c-\eta .
$$

(b) If the sequence $\left\{\alpha\left(m_{j}\right)\right\}_{j \in \mathbb{N}}$ does not tend to infinity, then we will find $x \in X$ such that the sequence $\left\{\left\|m_{j} x+a_{j}\right\|\right\}_{j \in \mathbb{N}}$ does not tend to 0 . We can suppose that $\lim _{j \rightarrow \infty}\left\|a_{j}\right\|=0$ (otherwise take $x=0$ ). There exist $M \in \mathbb{N}$ and an increasing sequence $\left\{j_{k}\right\}_{k \in \mathbb{N}}$ of natural numbers such that $\alpha\left(m_{j_{k}}\right)=M$ for all $k$. Find $N \in \mathbb{N}$ such that $2^{N}>M$ and $\psi(N) \leq c$. For $x=2^{-2^{N}}$ we get

$$
\sum_{n \in \mathbb{N}}\left\|2^{2^{n}} x\right\|=\sum_{n<N} 2^{2^{n}-2^{N}}=\psi(N) \leq c
$$

Since $\left\|m_{j_{k}} x+a_{j_{k}}\right\| \geq\left\|m_{j_{k}} x\right\|-\left\|a_{j_{k}}\right\| \geq 2^{M-2^{N}}-\left\|a_{j_{k}}\right\|$ for all $k \in \mathbb{N}$, the sequence $\left\{\left\|m_{j} x+a_{j}\right\|\right\}_{j \in \mathbb{N}}$ does not converge to 0 .

Proposition 11. For every $c>0$ there exists $X \subseteq \mathbb{T}$ such that

(1) for every $\eta>0$ there exists an increasing sequence of natural numbers $\left\{n_{k}\right\}_{k \in \mathbb{N}}$ such that $\sum_{k \in \mathbb{N}}\left\|n_{k} x\right\|<c+\eta$ for all $x \in X$;

(2) for every increasing sequence $\left\{m_{j}\right\}_{j \in \mathbb{N}}$ of natural numbers and for every sequence $\left\{a_{j}\right\}_{j \in \mathbb{N}}$ of elements of $\mathbb{T}$ there exists $x \in X$ such that $\sum_{j \in \mathbb{N}}\left\|m_{j} x+a_{j}\right\|$ $\geq c$.

Proof. Put

$$
X=\left\{x \in \mathbb{T}: \sum_{n \geq N}\left\|2^{2^{n}} x\right\| \leq c+\phi(N)+\psi(N) \text { for all } N \in \mathbb{N}\right\} .
$$

Clearly $X$ satisfies condition (1).

To show (2), fix sequences $\left\{m_{j}\right\}_{j \in \mathbb{N}},\left\{a_{j}\right\}_{j \in \mathbb{N}}$. We will find $x \in X$ such that $\sum_{j \in \mathbb{N}}\left\|m_{j} x+a_{j}\right\| \geq c$. Again let us discuss two cases.

(a) Assume that $\lim _{j \rightarrow \infty} \alpha\left(m_{j}\right)=\infty$. Let us choose a sequence $\left\{p_{k}\right\}_{k \in \mathbb{N}}$ of positive integers such that $\sum_{k \in \mathbb{N}} 2^{-2^{p_{k}}}=c$. Find $t \in \mathbb{N}$ such that $\left\|m_{0} 2^{-2^{t}}+a_{0}\right\|>$ 0 and put $\theta=\left\|m_{0} 2^{-2^{t}}+a_{0}\right\| / 2$. Fix a sequence $\left\{\varepsilon_{k}\right\}_{k \in \mathbb{N}}$ of positive reals such that $\sum_{k \in \mathbb{N}} \varepsilon_{k}=\theta$ and find $l \in \mathbb{N}$ such that $\alpha\left(m_{j}\right) \geq 2^{t}$ for all $j \geq l$. By Lemma 9 there exists $x^{\prime} \in \mathbb{T}$ such that

$\left(1^{\prime}\right)\left\|x^{\prime}\right\| \leq \theta / m_{0}$

$\left(2^{\prime}\right)$ there exists an increasing sequence $\left\{n_{k}\right\}_{k \in \mathbb{N}}$ of natural numbers such that $\left\|2^{2^{n_{k}}} x^{\prime}\right\| \leq 2^{-2^{p_{k}}}+2^{-2^{n_{k}}}$ for all $k \in \mathbb{N}$, and $\left\|2^{2^{n}} x^{\prime}\right\| \leq 2^{-2^{n}}$ for all $n \notin\left\{n_{k}\right.$ : $k \in \mathbb{N}\}$

$\left(3^{\prime}\right)$ there exists an increasing sequence $\left\{j_{k}\right\}_{k \in \mathbb{N}}$ of natural numbers such that $j_{k} \geq l$ and $\left\|m_{j_{k}} x^{\prime}+a_{j_{k}}\right\| \geq 2^{-2^{p_{k}}}-\varepsilon_{k}$ for all $k \in \mathbb{N}$. 
Put $x=x^{\prime}+2^{-2^{t}}$. Then for every $N \in \mathbb{N}$ we have

$$
\begin{aligned}
\sum_{n \geq N}\left\|2^{2^{n}} x\right\| & \leq \sum_{n \geq N}\left\|2^{2^{n}} x^{\prime}\right\|+\sum_{n \geq N}\left\|2^{2^{n}-2^{t}}\right\| \\
& \leq \sum_{k \in \mathbb{N}} 2^{-2^{p_{k}}}+\sum_{n \geq N} 2^{-2^{n}}+\sum_{N \leq n<t} 2^{2^{n}-2^{t}} \leq c+\phi(N)+\psi(N),
\end{aligned}
$$

since the last sum equals 0 if $t \leq N$ and is not greater than $\psi(t)<\psi(N)$ if $t>N$. Moreover,

$$
\begin{aligned}
\sum_{j \in \mathbb{N}}\left\|m_{j} x+a_{j}\right\| & \geq\left\|m_{0} x+a_{0}\right\|+\sum_{k \in \mathbb{N}}\left\|m_{j_{k}} x+a_{j_{k}}\right\| \\
& \geq\left\|m_{0} 2^{-2^{t}}+a_{0}\right\|-\left\|m_{0} x^{\prime}\right\|+\sum_{k \in \mathbb{N}}\left\|m_{j_{k}} x^{\prime}+a_{j_{k}}\right\| \\
& \geq \theta+\sum_{k \in \mathbb{N}} 2^{-2^{p_{k}}}-\sum_{k \in \mathbb{N}} \varepsilon_{k}=c .
\end{aligned}
$$

(b) If the sequence $\left\{\alpha\left(m_{j}\right)\right\}_{j \in \mathbb{N}}$ does not tend to infinity, then similarly as in the proof of Proposition 10 there exists $x \in \mathbb{T}$ such that $\sum_{n \in \mathbb{N}}\left\|2^{2^{n}} x\right\| \leq c$ and the sequence $\left\{\left\|m_{j} x+a_{j}\right\|\right\}_{j \in \mathbb{N}}$ does not tend to 0 .

The following theorem summarizes our results concerning the hierarchy of the families $\mathcal{B}_{0}^{c}$ and $\mathcal{B}_{0}^{* c}$.

Theorem 12. Let $c$ be a positive real. Then

$$
\bigcup_{d<c} \mathcal{B}_{0}^{d}=\bigcup_{d<c} \mathcal{B}_{0}^{* d} \varsubsetneqq \mathcal{B}_{0}^{c} \subseteq \mathcal{B}_{0}^{* c} \varsubsetneqq \bigcap_{d>c} \mathcal{B}_{0}^{d}=\bigcap_{d>c} \mathcal{B}_{0}^{* d} .
$$

Corollary 13. If $c<d$, then $\mathcal{B}_{0}^{c} \varsubsetneqq \mathcal{B}_{0}^{d}$ and $\mathcal{B}_{0}^{* c} \varsubsetneqq \mathcal{B}_{0}^{* d}$.

Let us note that the proofs of Propositions 10 and 11 can be easily adopted for showing the following statements.

Proposition 14. For every $c>0$ there exists $X \subseteq \mathbb{T}$ such that

(1) there exists an increasing sequence $\left\{n_{k}\right\}_{k \in \mathbb{N}}$ of natural numbers such that $\sum_{k \in \mathbb{N}}\left|\sin \pi n_{k} x\right| \leq c$ for all $x \in X$

(2) for every increasing sequence $\left\{m_{j}\right\}_{j \in \mathbb{N}}$ of natural numbers, every sequence $\left\{a_{j}\right\}_{j \in \mathbb{N}}$ of elements of $\mathbb{T}$, and for every $\eta>0$ there exists $x \in X$ such that $\sum_{j \in \mathbb{N}}\left|\sin \pi\left(m_{j} x+a_{j}\right)\right|>c-\eta$.

Proposition 15. For every $c>0$ there exists $X \subseteq \mathbb{T}$ such that

(1) for every $\eta>0$ there exists an increasing sequence of natural numbers $\left\{n_{k}\right\}_{k \in \mathbb{N}}$ such that $\sum_{k \in \mathbb{N}}\left|\sin \pi n_{k} x\right|<c+\eta$ for all $x \in X$;

(2) for every increasing sequence $\left\{m_{j}\right\}_{j \in \mathbb{N}}$ of natural numbers and for every sequence $\left\{a_{j}\right\}_{j \in \mathbb{N}}$ of elements of $\mathbb{T}$ there exists $x \in X$ such that

$$
\sum_{j \in \mathbb{N}}\left|\sin \pi\left(m_{j} x+a_{j}\right)\right| \geq c .
$$

Hence it is possible to prove results analogous to Theorem 12 and Corollary 13. concerning the families of sets obtained by replacing the expression $\sum_{k \in \mathbb{N}}\left\|n_{k} x\right\|$ in Definition 5 by $\sum_{k \in \mathbb{N}}\left|\sin \pi n_{k} x\right|$. 


\section{REFERENCES}

[1] J. Arbault, Sur l'ensemble de convergence absolue d'une série trigonométrique, Bull. Soc. Math. France 80 (1952), 253-317. MR 14:1080d

[2] L. Bukovský, N. N. Kholshchevnikova and M. Repický, Thin sets of harmonic analysis and infinite combinatorics, Real Anal. Exchange 20 (1994/95), 454-509. MR 97b:43004

[3] P. Eliaš, A classification of trigonometrical thin sets and their interrelations, Proc. Amer. Math. Soc. 125 (1997), 1111-1121. MR 97f:43006

Mathematical Institute, Slovak Academy of Sciences, Jesenná 5, 04154 Košice, SloVAKIA

E-mail address: elias@kosice.upjs.sk 